\title{
Infrasound Propagation of Finite Amplitude Wave in the Big Size Horn
}

\author{
Galyna Ivanovna Sokol, Vladyslav Yurievich Kotlov, Elena Sergeevna Mironenko, \\ Sergey Yurievich Kirichenko \\ Department of Physics and Technology, Dnipropetrovs'k National University named of Oles Gonchar, Dnepropetrovsk, Ukraine
}

\section{Email address:}

gsokol@ukr.net (G. I. Sokol), vlander8@gmail.com (V. Y. Kotlov), esinitsa09@gmail.com (E. S. Mironenko), kirichenkosergey@mail.ru (S. Y. Kirichenko)

\section{To cite this article:}

Galyna Ivanovna Sokol, Vladyslav Yurievich Kotlov, Elena Sergeevna Mironenko, Sergey Yurievich Kirichenko. Infrasound Propagation of Finite Amplitude Wave in the Big Size Horn. American Journal of Management Science and Engineering. Vol. 2, No. 5, 2017, pp. 117-122. doi: 10.11648/j.ajmse.20170205.15

Received: April 7, 2017; Accepted: April 21, 2017; Published: October 23, 2017

\begin{abstract}
The analytical expressions, allowing analyzing the laws of increase and decrease of the second harmonic at excitation of the finite amplitude wave in the air-horn, are obtained. The distance, where the second harmonic propagating along the air-horn reaches its maximum, is determined. Comparative analysis for air-horns of different shapes is performed. All calculations were made for the horn, whose length is 10 meters and the diameter of the outlet section 4 meters. The resonant frequency of the acoustic signal in such a mouthpiece is $20 \mathrm{~Hz}$.
\end{abstract}

Keywords: Finite Amplitude Wave, Air-Horn, Acoustic Signal, Infrasound Propagation, Big Size Horn, Sound Pressure, Amplitude of the Second Harmonic, Nonlinear Distortion Coefficients

\section{Introduction}

As a result of the sizeable increase of a loudspeaker's power there occurs the displacement of a membrane set in the trans-horn chamber. In this case the wave known in acoustics as a wave of finite amplitude is realized in the system [1-3]. Horns are considered in the following sources [4-6].

It propagates along the horn and the nonlinear acoustical effects have become significant at some distance from the horn throat. This appears in transferring of the energy part of a fundamental tone wave to the higher order harmonics $[7,8]$, the largest share of energy falling on the second harmonic. It is necessary to realize damping the second harmonic to guarantee effective emission on a major tone frequency.

Some methods of oscillations damping are known, which are applied in the technical systems with the conduits. The necessary effect is reached, for example, by installing a screen filter in the conduits and by lining a wall with a sound-absorbing material. But usually this task is solved without taking the nonlinear effects into consideration. In addition, the methods of oscillations damping in the conduits of a variable cross-section and in the horns, which would take account of their specific shape and exponent of extension, are insufficiently studied.

This work is aimed at studying changes of acoustic pressure amplitude of the second harmonic in the horn at excitation in horn throat of the finite amplitude wave as well as a determination of the coordinate of the horn's axial section at which the amplitude reaches its maximum value.

\section{Conditions of Nonlinear Effects' Development During Realization of a Plane Wave of Finite Amplitude}

The intensive sound waves differ from low-amplitude disturbances described in linear acoustic approximation [1-3] considerably. During the intensive wave propagation a gradual change of a waveform occurs as a result of the difference in motion speeds of its profile's different sections. Points referring to a bigger compression move faster, and as a result the steepness of compression fronts increases. Evolution of waveform can be rated as changing its spectral composition: the increase of the edges' steepness 
corresponds to high-frequency harmonics rising. A dissipation of wave energy and dispersion of its propagation speed opposes to the process of nonlinear increase of edges' steepness. An influence of dissipative effects - the viscosity and the thermal conduction - leads to wave profile smoothing, to decreasing velocity gradient and temperature gradient, to a faster damping of high-frequency components of waves spectrum. An influence of sound dispersion leads to the blurring of fronts' steepness as a result of the various harmonics "recession", which moves at different speeds. Therefore the propagation of intense acoustic excitation is defined by the competition of nonlinearity, dissipation and dispersion.

The comparative role of these factors is found out by means of searching the Korteweg-de Vries-Burgers equation [1-3].

The analysis of its solution for the case of a plane wave, propagating in the unlimited space, shows, that the nonlinearity generates the second harmonic. In case of absence of the dispersion the amplitude of second harmonic grows at first - reaching the peak at the distance $X_{\max }$ of

$$
\mathrm{X}_{\max }=\ln 2 /\left(2 \delta \omega^{2}\right)
$$

(here, $\delta$ is dissipation factor, $\omega$ is circular frequency) - and then it damps exponentially.

\section{The Nonlinear Oscillations in Horns at Propagation of the Wave of Finite Amplitude}

Often the occurrence of the nonlinear effects in system of sound transmission is concerned with the embodiment of a loudspeaker. For removing them, the special technical measures are taken in the mechanical part of a membrane suspension. Nonlinear distortions are also decreased by governing the irregularity of induction distribution in a gap [10]. Nonlinear distortions, connected with the magnetic field nonuniformity in a magnetic circuit's gap and violation of Hooke law, are not so substantial. Factors, related to nonlinear effects in a horn and in the chamber ahead of a horn [7], are more substantial.

At the same time, although the membrane executes a simple harmonic vibration, the wave's propagation does not already go by the sine law, and there are both fundamental harmonic and the harmonics of higher orders [8] in it. In the well-known loudspeakers constructions, the elements decreasing or removing the nonlinear effects in a horn completely were not provided. This decreased the efficiency of fundamental tone's sound generation.

In the systems of sound transmission and in the broadcasting systems the horns of different shapes are used extensively, in that way the active component of acoustic power is increased significantly [7-10].

The exact shape of a horn determinates the law of change of cross-sectional area. The longitudinal sections of the most often occurring horns are depicted in Figure 1.

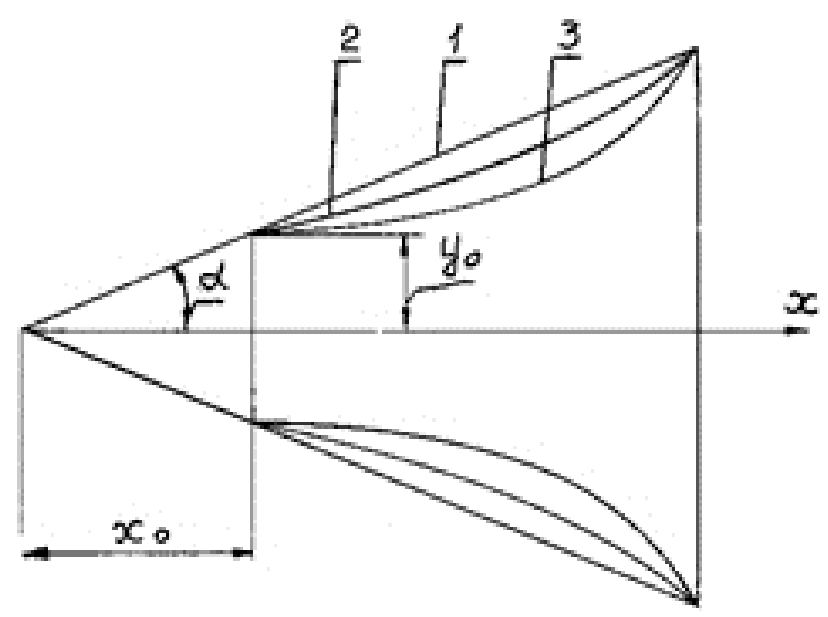

Figure 1. The shapes of horns: 1: the conical shape; 2: the exponential shape; 3: the catenoid shape; $x_{0}, y_{0}$ are coordinates of the initial crosssection of a horn throat; $a$ is apex angle.

\subsection{The Exponential Horn}

The sectional area of an exponential horn is measured by such a law

$$
\mathrm{Sx}=\mathrm{S}_{0} \mathrm{e}^{\beta \mathrm{x}}
$$

where $S_{o}$ is the sectional area of a horn throat, $\beta$ is the index of extension per one meter.

The sound pressure in each harmonics propagating along the axis of the horn is described by equation, binding the value of sound pressure $(p)$ in any point $(x)$ along the longitudinal axis $O X$ of the horn, the peak value of sound power in the horn throat $\left(p_{m}\right)$ and the exponent of the horn extension $(\beta)$.

To the horn exponential form

$$
p=p_{m} \exp (-0,5 \beta x)
$$

To the first and second harmonics the equation (1) is written in the form of

$$
p_{i}=p_{m i} \exp (-0,5 \beta x)
$$

Let us consider the process of changing of the sound pressure amplitude in the second harmonic $p_{2 \Sigma}$ along the axis of the horn not only at the expense of axial section changing, but also at the expense of nonlinear effects' appearing.

The theory of wave of finite amplitude lets us know that as range of the wave the amplitude of second harmonic is changed by the law

$$
p_{2}=C \cdot p_{1}^{2} \cdot x, C=\frac{1}{4} \cdot \frac{\gamma+1}{\gamma \cdot P_{0}} \cdot \frac{\varpi}{c_{0}} .
$$

$C$ is parameter, $\gamma$ is specific gravity of air, $p o$ is air pressure, $\omega$ is circular frequency, $c o$ is speed of sound in a medium.

In accordance with technique, suggested by V. V. Furdujev in his work [6] we can define $p_{2 \Sigma}$ as:

$$
p_{2 \Sigma}=\frac{2 C \cdot p_{m 1}^{2}}{\beta}[\exp (-0,5 \beta x)-\exp (\beta x)]
$$


The rate of change of the second harmonic amplitude is solved by the equation

$$
\frac{d p_{2}}{d x}+\frac{\beta}{2} \cdot p_{2}=C \cdot p_{m 1}^{2} \exp (-\beta x)
$$

The equation (4) is solved in the work [8] concerning $p_{2}$, and the expression is found, which shows the change of the second harmonic amplitude along a horn of exponential form:

$$
p_{2 e x}=\frac{2 c_{m 1}^{2}}{\beta} \exp \left(-\frac{\beta x}{2}\right)-\left(p_{m 1} \cdot \exp \left(-\frac{\beta x}{2}\right)\right)
$$

\subsection{The Horn in a Catenoid Shape}

In sound's broadcasting horns of the exponential form are commonly used for the reproduction of high frequencies. Now the attention is paid to the efficient reproduction in the low-frequency region. In this case using of horns of catenoid shape is more profitable [9].

Let a wave of finite amplitude with quantity of sound pressure $p_{2 \Sigma}$ be realized in a horn throat. The walls of the horn are considered absolutely stiff in comparison with the air quality filing it. Then we are finding the expression for the nonlinear distortion factor $v_{c a t}$ and for the second harmonic amplitude $p_{2 c a t}$ in case of horn in form of a catenoid Changing the sectional area $S_{x}$ in the horn in catenoid shape occurs by the law

$$
S_{x}=S_{0} \cdot c h^{2}(\beta x) .
$$

The sound pressure amplitude of the first harmonic $p_{\text {lcat }}$ and the second harmonic $p_{2 c a t}$ along the horn in catenoid shape is changing along the axes according to the relations

$$
\begin{gathered}
p_{1 \text { cat }}=p_{m 1} / \operatorname{ch}(\beta x), \\
p_{2 c a t}=p_{m 2} / \operatorname{ch}(\beta x), I=1,2 .
\end{gathered}
$$

Changing the sound pressure of the second harmonic $p_{\text {Icat }}$ at small value in the horn throat $p_{m 1}$ is depicted in Figure 2 .

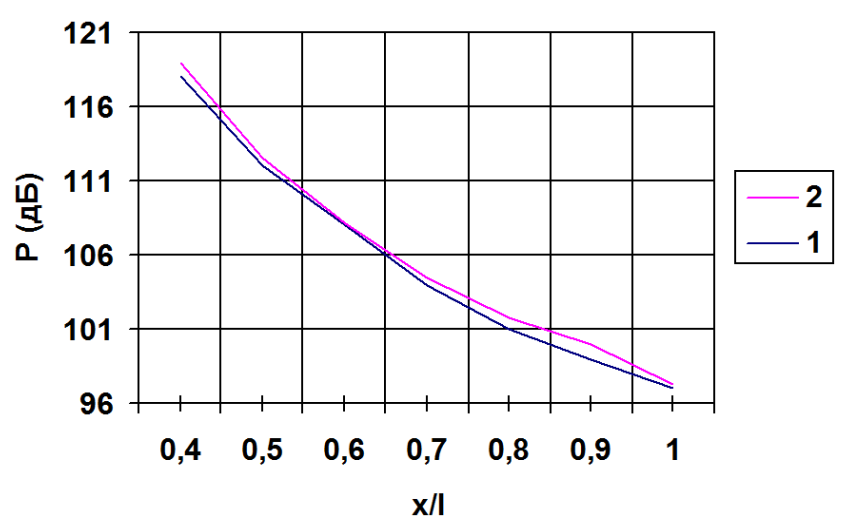

Figure 2. Changing the first harmonic along the axes of a horn. 1: the theoretical curve; 2: the experimental values.

Let us write the expression for the rate of change of the second harmonic $d p_{2 c a t} / d x$.

$$
\frac{d p_{2 c a t}}{d x}=C \cdot p_{1 c a t}^{2}-\beta t h(\beta x) \cdot p_{2 c a t}
$$

This result in the following

$$
\frac{d p_{2 c a t}}{d x}+\beta \operatorname{th}(\beta x) \cdot p_{2 c a t}=C \cdot p_{m 1}^{2} / \operatorname{ch}^{2}(\beta x) .
$$

The equation (7) is the first-order non-homogeneous linear differential equation. The general solution is in the form

$$
p_{2 c a t}=\frac{2 C \cdot p_{m 1}^{2} \operatorname{arctg} \exp (\beta x)}{\beta \operatorname{ch}(x \beta)}+\frac{D}{\operatorname{ch}(\beta x)}, D-\text { const } .
$$

As the second harmonic appears only during the propagation of wave the solution of equation should satisfy the boundary condition

$$
p_{2 c a t}=0 \text { by } x=0 .
$$

The particular solution of the equation (7) satisfying the mentioned conditions has a form

$$
p_{2 c a t}=\frac{2 c \cdot p_{m 1}^{2}}{\beta \operatorname{ch}(x \beta)} \cdot\left[\operatorname{arctg} \exp (\beta x)-\frac{\pi}{4}\right] .
$$

Formula (9) implies the expression for the nonlinear distortion factor $v_{\text {cat }}$ :

$$
v_{c a t}=2 C \cdot p_{m 1}^{2} \cdot\left[\operatorname{arctg} \exp (\beta x)-\frac{\pi}{4}\right] / \beta .
$$

\subsection{The Conic Horn}

Let us consider the horn of conical shape. The analytical dependence characterizing the depreciation of amplitude $p i$ any harmonic along the axes of the conical shape horn has a form:

$$
p_{1}=p_{m 1} \cdot \frac{x_{0}}{x+x_{0}}, p_{2}=p_{m 2} \cdot \frac{x_{0}}{x+x_{0}}, I=1,2 .
$$

where $p_{m i}$ is amplitude in the horn throat, xo is the distance from throat to the cone point (Figure 1).

The second harmonic amplitude as wave is ranging at a conical horn goes up with the rate

$$
\frac{d p_{i}}{d x}=-p_{m i} \cdot \frac{x_{0}}{\left(x+x_{0}\right)^{2}}=-p_{i} \cdot \frac{1}{\left(x+x_{0}\right)},
$$

Recording the total rate of second harmonic amplitude change in conical horn (subject to nonlinear effects) results in the non-homogeneous linear differential equation

$$
\frac{d p_{2}}{d x}=C \cdot p_{m 1}^{2} \cdot \frac{x_{0}^{2}}{\left(x+x_{0}\right)^{2}} .
$$

Having solved it, we will find the analytic dependence describing the change of second harmonic amplitude during its propagation along the conical horn subject to nonlinearity.

$$
p_{2 c o n}=\frac{c \cdot p_{m 1}^{2} \cdot \ln \left(x+x_{0}\right) \cdot x_{0}^{2}}{x+x_{0}}+\frac{D}{x+x_{0}}, D-\text { const } .
$$

The unknown arbitrary constant $D$ is found, satisfying the equation (12) solution (13) for the boundary condition: 


$$
p_{2 \text { con }}=0 \text { by } x=0 \text {. }
$$

It is easy to verify that particular solution of the equation (12) subjecting to boundary condition has a form:

$$
p_{2 \text { con }}=C \cdot p_{m 1}^{2} \frac{x_{0}^{2}}{\left(x+x_{0}\right)} \cdot \ln \left(\frac{x+x_{0}}{x_{0}}\right) .
$$

Using the derived expression, let us record the formula for the nonlinear distortion factor $v_{\text {con }}$ :

$$
v_{\text {con }}=\frac{p_{2 \operatorname{con}(x)}}{p_{1 \operatorname{con}(x)}(x)} \cdot p_{m 1}^{2} \cdot \ln \left(\frac{x+x_{0}}{x_{0}}\right) .
$$

The comparison with the nonlinear distortion factors in horns of exponential shape and horns in catenoid shape shows that with increasing of the coordinate $\mathrm{x}$ in horn of conical shape $v_{\text {con }} \rightarrow$, but this adversely affected work of an audio oscillator supplied with horns of such pattern.

\section{The Determining of Positions of Second Harmonics Peaks Xmax for Different Horns Shapes}

To the determination $x_{\max }$ of distance from the horn throat to the point, where the second harmonic of sound pressure reaches its peak, we will use the necessary extreme condition [11].

\subsection{The Exponential Horn}

According to relation (5) we have the following formula for second harmonic amplitude of sound pressure in horn of exponential shape:

$$
p_{2 e x}=\frac{2 C \cdot p_{m 1}^{2}}{\beta} \exp \left(-\frac{\beta x}{2}\right)-\frac{2 C}{2} \cdot\left(p_{m 1} \exp \left(-\frac{\beta x}{2}\right)\right)^{2}
$$

Having calculated the first derivative of function of second harmonic amplitude and having equaled it to zero, we will determine the distance $x_{\max }$ from the horn throat to the peak of the second harmonic:

$$
\frac{d p_{2 e x}}{d x}=C \cdot p_{m 1}^{2}[-\exp (-0,5 \beta x)+2 \exp (-\beta x)]=0 .
$$

As $C p^{2}{ }_{m l} \neq 0$, let us analyze the expression

$$
2 \exp (-\beta x)-\exp (-0,5 \beta x)=0,
$$

where we have derived the following after taking the logarithm

$$
x_{\max }=(2 \ln 2) / \beta .
$$

As the second derivative of function (17) is equal

$$
\frac{d^{2} p_{2 e x}}{d x^{2}}=C \cdot p_{m 1}^{2} \cdot \beta(0,5 \exp (-0,5 \beta x)-2 \exp (-\beta x)),
$$

and possesses the negative value in the $x_{\max }$ point, the function of the second harmonic amplitude of the sound pressure obtains its peak in this point.

$$
p_{2 e x}^{*}=\frac{C \cdot p_{m 1}^{2}}{2 \beta}
$$

\subsection{The Catenoid Horn}

Having equaled the first derivative of function described by the expression (9) to zero we will obtain

$$
\frac{d p_{2 c a t}}{d x}=\frac{c \cdot p_{m 1}^{2}}{c^{2}(\beta x)}\left\{\frac{1}{2}-(\operatorname{arctg}(\exp (\beta x))-\operatorname{arctg} 1) \operatorname{sh}(\beta x)\right\}=0 .
$$

As $C \cdot p_{m 2}^{2} / c h^{2}(\beta x) \neq 0$ whatever value of $\mathrm{x}$ is we will equal the second multiplier to zero to finding out the $\mathrm{X} \max$

$$
\frac{1}{2}-(\operatorname{arctg}(\exp (\beta x))-\operatorname{arctg} 1) \operatorname{sh}(\beta x)=0 .
$$

We will expand the function $\operatorname{arctg}(\exp (\beta x))$ into series. It is showed, that type of such expansion depend on if its argument will be greater or less then one. Obviously, that $\exp (\beta x)<1$, at $\beta x<0$. This condition is inadmissible to the problem of waves propagation in horn, as $x \geq 0$ and $\beta>0$. Therefore we will analyze the case $\exp (\beta x) \geq 1$.

Then, according to [11], the function $\operatorname{arctg} \exp (\beta x)$ is expanding into series in the following way:

$$
\operatorname{arctg} \exp (\beta x)=\frac{\pi}{2}-\frac{1}{\exp (\beta x)}+\frac{1}{[3 \exp (3 \beta x)]}-\cdots
$$

The expression in the right side of the formula (20) is alternating series. Solving the equation (19), we will be limited by the two first summands of the expansion (20). According to a well-known property of alternation series [11], the absolute magnitude error appearing in this case will not rank over the first neglected term (in our case $1 / \exp (3$ $\beta x)$ ).
Based on this, we will record the equation (19) in the form:

$$
\left(\frac{\pi}{4}-\exp (-\beta x)\right)(\exp (\beta x)-\exp (-\beta x))=1
$$

Having entered the designation $y=\exp (\beta x)$, we will derive the nonlinear equation:

$$
\left(\frac{\pi}{4}-\frac{1}{y}\right) \cdot\left(y-\frac{1}{y}\right)=1
$$

Allowing, that $y \neq 0$, we will come to the cubic equation

$$
\pi y^{3}-8 y^{2}-\pi y+4=0,
$$

studying which, we deduce, that it has three real roots, contained in the ranges of $(-1,-0.15),(0,1),(2,3)$. As we deal with values $y>1$, we will search the solution of equation (22) on the segment $(2,3)$. By means of Newton's method (or method of tangent lines) we determine the sought root $y^{*}=2,741832$ accurate within 10-10.

Consequently, the peak position of the sound pressure's second harmonic concerning catenoid shape horn throat is defined as: 


$$
x_{\max }=\frac{1}{\beta} \cdot \ln (2,741832) .
$$

The peak value of the second harmonic amplitude is equal to:

$$
p_{2 c a t}^{*}=\frac{0,733 c \cdot p_{m 1}^{2}}{\beta}
$$

\subsection{The Conic Horn}

Let us find out $\mathrm{X}$ max for the conical shape horn. The derivative of function of the sound pressure's second harmonic $p_{2 \text { con }}(x)$ represented by formula (15) after transformation has the form:

$$
\frac{d p_{2}}{d x}=C \cdot p_{m 1}^{2} \cdot\left[1-\ln \left(\frac{x}{x_{0}}+1\right)\right] /\left(x+x_{0}\right)^{2} .
$$

Obviously, given $x=x_{0}$, the analyzed derivative goes to infinity just as function itself. Therefore, $x=x_{0}$ is the minimal coordinate.

To the defining of $x_{\max }$ we have the equation:

$$
1-\ln \left(\frac{x}{x_{0}}+1\right)=0
$$

This result in the following

$$
x_{\max }=1,718 x_{0} .
$$

As $x_{0}=y_{0} / \operatorname{tg} \alpha$, then

$$
x_{\max }=1,718 y_{0} / \operatorname{tg} \alpha .
$$

We can simply see, that the bigger the horn's apex angle is, the closer to the horn throat the nonlinear effects appear. We have to point for completeness that the second harmonic amplitude's peak value is equal to:

$$
p_{2 c o n}^{*}=\frac{C \cdot p_{m 1}^{2} \cdot \operatorname{tg} \alpha}{2,718 y_{0}}=\frac{C \cdot p_{m 1}^{2}}{2,718 x_{0}}
$$

\section{The Comparative Analysis of the Second Harmonic Amplitudes Change}

In Figure 3 the curves showing the second harmonic's change in horns of exponential, conic and catenoid shapes are depicted.

The calculations of the second harmonics' curves were made for the following initial data: the velocity of sound in medium $c_{0}=340 \mathrm{M} / \mathrm{sec}$, exponent of the horn extension $\beta=$ 0.63 , the critical frequency $f_{c r}=17 \mathrm{~Hz}$, the circular frequency $\omega_{0}=106.8 \mathrm{rad} / \mathrm{c}$, the displacement amplitude in the horn throat $\xi=0.05 \mathrm{M}$, the major tone's initial amplitude in the horn throat $p_{m l}=2.2 \mathrm{kPa}$. The curves were constructed on the frequencies $f=17 \mathrm{~Hz}, 20 \mathrm{~Hz}, 23 \mathrm{~Hz}, 26 \mathrm{~Hz}, 29 \mathrm{~Hz}$.

It is clear from the graph that for the horns of exponential and catenoid shapes the peaks are clear retraced. There is no clear peak for the horn of conical shape. And at the same time, in horns of exponential and catenoid shapes values of second harmonic verge toward zero in the horn's orifices, but in a conical shape horn the second harmonic, approaching to the orifices, decreases very lightly.

We should notice that $x_{\max c a t}<x_{\max e x}$, i.e. the second harmonic in catenoid shape horn reaches its peak value more rapidly. In addition, for the same working frequencies and at the identical particle displacements in a horn throat the second harmonic amplitude's peak value is 1.25 times as big as in the catenoid shape horn, then in the horn of exponential shape.

All calculations were made for the horn, whose length is 10 meters and the diameter of the outlet section 4 meters. The resonant frequency of the acoustic signal in such a mouthpiece is $20 \mathrm{~Hz}$.

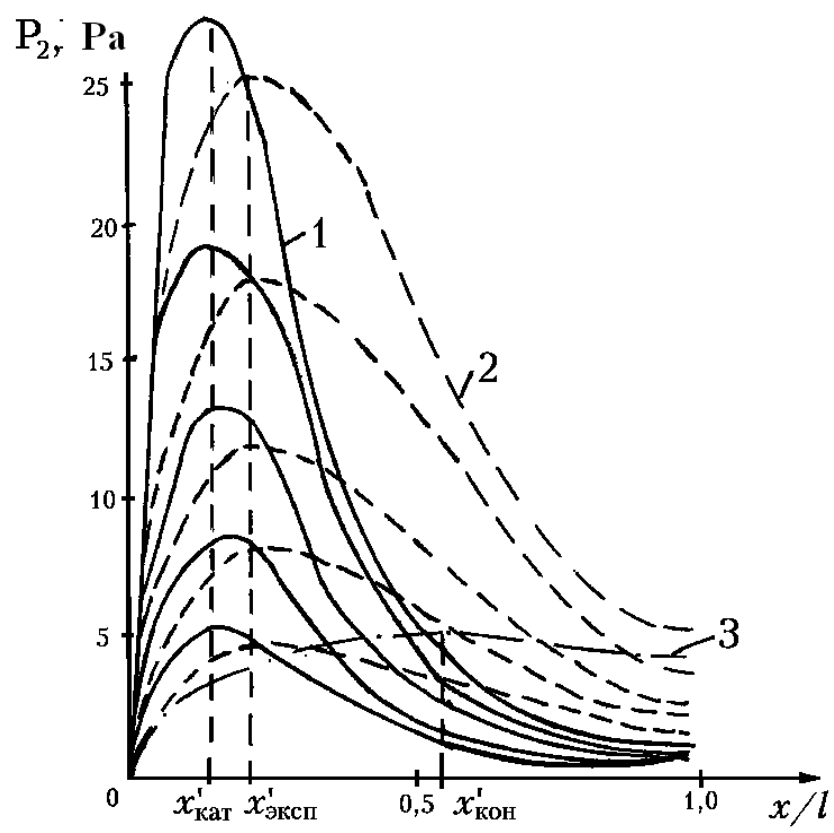

Figure 3. Graph of the second harmonic amplitudes' change along the horns. 1: the catenoid shape of horn; 2: the exponential shape of horn; 3: the conical shape of horn, $x^{\prime}=X_{\max }$ is the coordinate of horn's axial section, $l$ is its finite length.

\section{The Comparative Analysis of Nonlinear Distortion Factors Change}

We will assay the effect of damping, the nonlinear distortion factor $v$, which is defined as a relation of the first and second harmonics' amplitudes. In particular, in the work [8] the expression for nonlinear distortion factor in case of finite amplitude wave propagation in the exponential shape horn is found. The graphs of nonlinear distortion factors $v_{\text {exp }}, v_{\text {cat }}$ and $v_{\text {con }}$, depicted in Figure 4, for horns of exponential, catenoid and conical shapes on emission frequency $f=17 \mathrm{~Hz}, \xi=0,05 \mathrm{~m}$, were calculated on the basis of the expressions (10) and (16), where $\boldsymbol{v}_{0}$ is the limit value of nonlinear distortion factor. For the exponential shape horn it is equal to $2 C \cdot p_{m 1} / \beta$, for the catenoid shape horn it is $v_{0}=\pi \cdot C \cdot p_{m 1} / 2 \beta$. At the same time, $v_{c a t} / v_{\text {exp }}=$ $\left[\operatorname{arctg} \exp (\beta x)-\frac{\pi}{4}\right] /[1-\exp (-0,5 \beta x)]$ is true for the factors $v_{\text {exp }}, v_{\text {cat }}$. 
The nonlinear distortion factor $v$ con for the conical shape horn increases at the horn extension while for the horns of exponential and catenoid horns the factors $v_{\text {exp }}$ and $v_{\text {cat }}$ tend to the certain limit value.

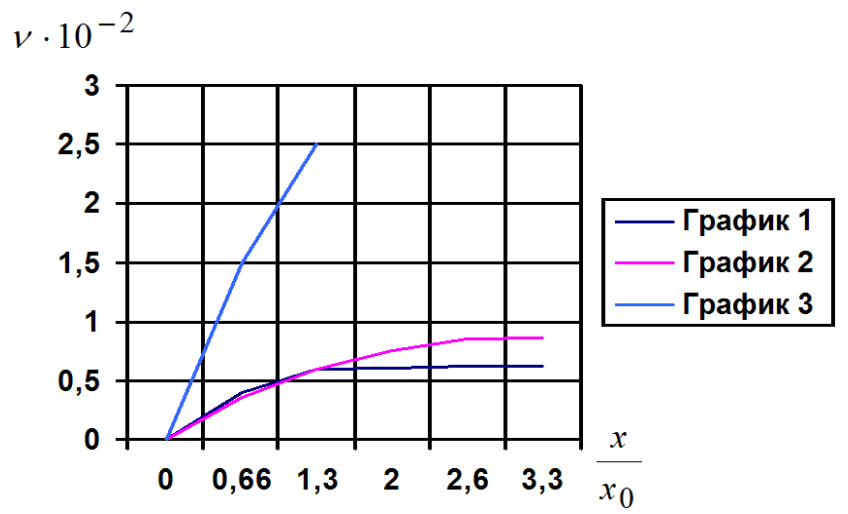

Figure 4. The nonlinear distortion factors changer. 1: the graph of nonlinear distortion factor $v_{\text {con }}, 2$ : the graph of nonlinear distortion factor $v_{e x}, 3:$ the graph of nonlinear distortion factor $v_{\text {cat }}$.

\section{Conclusion}

1. The equations have been received, describing the amplitude of the second harmonic's sound pressure and it's analog of velocity of propagation in horns of catenoid and conical shapes.

2. The analytical expressions have been obtained, allowing defining the coordinate $x_{\max }$ of cross-section, where the sound pressure's second harmonic reaches its peak, for the horns of conical, exponential and catenoid shapes.

3. The expressions have been derived, defining the nonlinear distortion coefficients $v$ for the horns of three shapes.

\section{References}

[1] Naugol'nykh K. A. Ostrovskiy L. A. Nonlinear wave processes in acoustics. M.: - Nauka, 1990. - 237 p.

[2] Zarembo L. K., Krasil'nikov V. A. Introduction to nonlinear acoustics. - M.: Nauka, 1966, 335 p.

[3] Atchley A. A. Not your ordinary sound experience: a nonlinear-acoustic primer / Acoustic Today, Vol. 5, ISSUE 1, October 2005. - P. $19-24$.

[4] Yue $\mathrm{Hu}$, Xilu Zhao, Takao Yamaguchi, Manabu Sasajima, Tatsushi Sasanuma, and Akira Hara, Effects of the Cone and Edge on the Acoustic Characteristics of a Cone Loudspeaker, Advances in Acoustics and Vibration Volume 2017 (2017), Article ID 2792376, 12 pages.

[5] Schneider H., Agerkvist F., Knott A., Andersen M. A. E. (2015), Investigation of Current Driven Loudspeakers, Dissertation presented at the 138th Convention of the Audio Engineering Society, Warsaw.

[6] T. Lukkari, J. Malinen, A posteriori error estimates for Webster's equation in wave propagation, Journal of Mathematical Analysis and Applications 427 (2) (2015) 941961.

[7] Vakhitov Ya. M. Theoretical foundations of acoustics and electroacoustic devices. - M.: Iskusstvo, 1982. - 400 p.

[8] Furduev V. V. Electroacoustics. - M.: GTTI, 1948. - 256 p.

[9] Morz F. Vibrations and sound. - M.: IIL, 1948. - 660 p.

[10] Sapozhkov M. A. Electroacoustics. - M.: Svyaz', 1978. - 275 p.

[11] Bronshteyn I. N., Semendyaev K. A. Handbook of mathematics for engineers and students. - M.: Nauka, 1986. - 554 p. 